\title{
Trabajo, fecundidad y condición femenina en México
}

\author{
Orlandina de Oliveira* \\ Brígida García**
}

\begin{abstract}
En este artículo se analizan inicialmente los cambios ocurridos en el trabajo femenino y sus condicionantes durante ios años de recesión económica de la década de ios ochenta. La información proviene de algunas de las principales encuestas de fecundidad lievadas a cabo durante ese periodo. Se hace hincapié en el aumento observado en la participación económica de mujeres de mayor èdad, unidas y con hijos, y en lás modificaciones que esto trae para las reiaciones conocidas entre fecundidad y trabajo. En una segunda parte se expioran las diversas formas en que las mujeres posiblemente viven ios cambios observados y el impacto esperado sobre ia condición de subordinación que las caracteriza. No se trata en este caso de resuitados de investigación, sino de una revisión bibliográfica, acompañada de planteamientos teórico-metodoiógicos sobre ia mejor manera de acercarse a este importante objeto de estudio.
\end{abstract}

Durante las últimas 4 décadas, la sociedad mexicana ha experimentado transformaciones económicas y sociodemográficas considerables; la acelerada urbanización, la ampliación de la industrialización, la acentuada migración rural-urbana, la expansión del sistema educativo y la diversificación de los mercados de trabajo urbanos, son algunos de los aspectos que caracterizan este rápido proceso de cambio social. Entre las transformaciones más sobresalientes que atañen a las mujeres mexicanas están su creciente participación en el mercado de trabajo y el descenso en la fecundidad que se inicia en los años setenta, debido a la expansión en el uso de anticunceptivos femeninos.

Las diferentes dimensiones de cambio señaladas han evolucionado a ritmos distintos y es necesario ampliar nuestro conocimiento del impacto de la recesión económica de los años ochenta sobre cada una de ellas. Como es conocido, después de 3 décadas de estabilidad, México experimenta en los años setenta periodos de recesión y recuperación parcial que truncan el desarrollo esta-

\footnotetext{
* Directora del Centro de Estudios Sociológicos dé El Colegio de México.

** Profesora-investigadora del Centro de Estudios Demográficos y de Desarrollo Urbano de El Colegio de México.
} 
bilizador y llevan a una transición hacia un patrón distinto de organización económica. La década de los ochenta, que se inicia con un pasajero repunte económico impulsado por el petróleo, se caracteriza desde 1982 por una severa y prolongada crisis que hasta hoy nos afecta. Son indicadores suficientemente conocidos de este fenómeno: la caída del producto por persona y del salario real de los trabajadores, la devaluación de la moneda y la inflación elevada en una parte importante del periodo. Estos aspectos, aunados a la deficiencia en los sistemas de seguridad social, y a la reducción de los subsidios otorgados a los productos básicos, han traído como consecuencia en México, al igual que en otros países de América Latina, un claro deterioro en los niveles de vida de la población (véanse Lustig, 1986; Casar y Ros, 1987; Rendón y Salas, 1987; Tello, 1979 y 1987). La crisis económica ha hecho más visible el carácter desigual y excluyente del desarrollo mexicano que ya en sus años de expansión se caracterizaba por concentración de la riqueza y por la persistencia de fuertes desigualdades sociales.

En este artículo presentamos inicialmente algunas evidencias sobre los principales cambios ocurridos en el trabajo femenino y sus condicionantes en los años de recesión económica. ${ }^{1}$ En un momento posterior describimos brevemente las principales características del descenso en la fecundidad mexicana, para luego hacer hincapié en la relación entre fecundidad y trabajo antes y después de los años de recesión en diferentes sectores sociales. En una última parte, presentamos algunas líneas de investigaión e hipótesis acerca de las repercusiones de las transformaciones señaladas sobre la condición social de las mujeres mexicanas.

La marcada expansión del trabajo femenino en los años de crisis

La presencia femenina en la actividad económica en México ha experimentado un aumento continuo en las últimas décadas: en $195013 \%$ de las mujeres de 12 años y más se declararon como eco-

${ }^{1}$ Los resultados aquí presentados son parte de una investigación más amplia sobre trabajo, fecundidad y condición femenina en México que realizan las autoras. Los datos que respaldan las distintas aseveraciones provienen de 3 encuestas de fecundidad llevadas a cabo en el país (Encuesta Mundial de Fecundidad, 1979; Encuesta Nacional Demográfica, 1982; Encuesta Nacional sobre Fecundidad y Salud, 1987), y se presentan y analizan en detalle en García y Oliveira (1990 y 1990a). Para mayor información remitimos al lector a esos textos inéditos. 
nómicamente activas; esta cifra fue de $16 \%$ en 1970 ; de $21 \%$ aproximadamente en 1979; 25\% en 1982 y 32 en 1987 (Oliveira y García, 1990; Pedrero, 1990).

Hasta principios de los años setenta, la mayoría de las mujeres que trabajaban fuera de su casa eran jóvenes y solteras o sin hijos. Conforme a nuestros análisis, durante las 2 décadas siguientes esta situación se ha visto sustancialmente modificada: entre 1976 y 1987, se registra con claridad un importante incremento en la participación económica de las mujeres unidas y de mayor edad.

La recesión económica de la década de los ochenta se señala a menudo como un factor que ha incidido en el aumento reciente de la participación económica femenina, aunque ciertamente por motivos distintos a los observados en épocas de auge donde se ponía el acento en la modernización de la economía y la ampliación de ocupaciones femeninas no manuales.

Indiscutiblemente, el deterioro de los niveles de vida en estos años ha hecho cada vez más indispensable para la población de bajos y medianos ingresos la utilización de los diversos mecanismos de organización familiar de la reproducción cotidiana. En este contexto las mujeres amplían su participación en el mercado para contribuir a la satisfacción de las necesidades básicas de los hogares (Selva, 1985; Cortés, 1988; Pacheco, 1988; González de la Rocha, 1989; Oliveira, 1989).

Nuestros resultados dejan claro que la recesión económica ha llevado a la movilización de una oferta potencial de mano de obra constituida principalmente por mujeres unidas con hijos, las cuales salen al mercado en busca de un trabajo o crean su propio empleo. También encontramos que aumenta la participación económica de las mujeres sin escolaridad o con primaria incompleta entre 1982-1987. Por su parte, las mujeres jóvenes de mediana y alta escolaridad, no incrementan su presencia relativa en el mercado de trabajo en el mismo periodo.

Estos hallazgos sobre mayores limitaciones en el ritmo de incremento de las oportunidades de trabajo para las mujeres jóvenes nos apartan visiblemente de otros momentos del acontecer nacional e internacional y de las realidades de países desarrollados. Para estas otras situaciones está suficientemente documentado que un nivel de escolaridad mayor se asocia a un nivel de participación elevado y creciente a lo largo del tiempo (véanse, por ejemplo, Pedrero y Rendón, 1982; Wainerman y Recchini, 1981; Blau y Ferber, 1985).

El marcado incremento en la participación económica de las mujeres con baja escolaridad, unidas y con hijos chicos, ha estado acompañado de modificaciones importantes en las ocupaciones 
que se desempeñan. Las actividades manuales por cuenta propia se han constituido en fuente importante de actividad para amplios sectores de mujeres. Para el conjunto del país nuestros datos indican que entre 1982 y 1987 las ocupaciones manuales no asalariadas pasan de 7.6 a $18.5 \%$ de las actividades que desempeñan las mujeres de 20 a 49 años. Esto es principalmente cierto para las trabajadoras con escolaridad mínima, las unidas, en particular aquellas con más de 3 hijos, y las separadas, divorciadas y viudas.

La expansión relativa de las trabajadoras por cuenta propia se registra en algunas regiones del país desde los años setenta $\mathbf{y}$ también se manifiesta en años recientes en algunas de las principales ciudades ubicadas en el centro y en el sureste mexicanos. $\mathrm{Si}$ se toma en cuenta el peso cuantitativo de estas ciudades, tal parecería que en el periodo 1984-1987, el aumento de la actividad de las mujeres se debe, en importante medida, al incremento del trabajo por cuenta propia (Oliveira, 1989).

Dicho incremento se ha dado no sólo en el terciario - contexto económico donde tienen mayor peso- sino en la industria. El desglose de las actividades manuales por cuenta propia pone de manifiesto que durante los años de recesión se ampliaron por un lado, las actividades de autoempleo vinculadas directamente con la mayor pobreza relativa de los hogares. Éste es probablemente el caso de gran parte de las vendedoras ambulantes que prácticamente doblaron su peso relativo de 1982 a 1987 y son uno de los efectos más visibles de la crisis de los años ochenta. Por el otro lado, están las actividades de producción por cuenta propia que, al igual que las actividades de venta ambulante, duplicaron su importancia relativa en los ochenta.

\section{Algunos aspectos del descenso de la fecundidad}

La marcada reducción en la fecundidad - resultado del proceso de modernización del país y de la política de planificación familiar- que se inicia a mediados de los setenta, está bien documentada en la literatura. La tasa global que refleja el número final de hijos que tendrían las mujeres si se conservara el patrón de fecundidad del momento, era de 6.3 en 1973 y pasó a 3.8 en 1986. La reducción fue más marcada al inicio de la baja que en años posteriores (Palma, 1988).

Las prácticas de control natal han tenido amplia incidencia en la explicación de la disminución de la fecundidad en el país. La aceptación de métodos anticonceptivos se ha incrementado en forma notable a partir de mediados de los setenta: el porcentaje 
de mujeres unidas que los usan pasó de 30.2 en 1976 a 52.7 en 1987 (Bronfman, López y Tuirán, 1986; Cervantes y Sandoval, 1988).

El cambio en la conducta reproductiva ha sido observado entre mujeres de diferentes edades pero con intensidades distintas. Las de mediana edad (35 a 39 años) fueron las primeras en bajar su fecundidad; en un segundo momento siguen las más jóvenes (15-19 años) mientras que las mujeres en las edades de mayor reproducción (20 a 34) han presentado el menor descenso a lo largo del periodo analizado, sobre todo las de 20 a 24 años (datos de Palma, 1988).

Los ritmos distintos de reducción de la fecundidad entre grupos, aunados a los niveles iniciales también diversos, han contribuido a disminuir la distancia entre sectores sociales. Sin embarge; los diferenciales aún son marcados, pues las mujeres pertenecientes a los sectores más favorecidos son las de fecundidad más baja mientras las de los sectores agrícolas más pobres tienen los niveles más elevados (Mier, Terán y Rabell, 1984; López y Tuirán, 1983; García, B., 1987; García, I., 1989).

Estos resultados denotan las profundas desigualdades sociales que existen en la sociedad mexicana que permean desde las condiciones materiales de existencia hasta las propias alternativas abiertas a los individuos para reproducirse biológicamente. También para entender las variaciones en el descenso de la fecundidad entre los diversos sectores de la sociedad y entre generaciones distintas de mujeres hay que tener en cuenta las costumbres, los valores y los patrones de conducta compartidos o no por grupos de individuos que tienen experiencias de vida similares.

\section{Cambios en la relación entre fecundidad y trabajo}

El aumento en la participación económica de las mujeres unidas nos ha llevado a retomar en otros estudios, la amplia y compleja discusión sobre la influencia que ejerce el trabajo de la mujer sobre su fecundidad. La síntesis a la que llegamos pone en evidencia que muchos de los análisis recientes para países no desarrollados muestran más bien que el número de hijos condiciona el tipo de trabaje que las mujeres desempeñan en vez de la relación inversa (véanse Naciones Unidas, 1987; García y Oliveira, 1989).

En México, la influencia inhibidora de los hijos sobre el trabajo femenino es clara; nuestros datos para mujeres de 20 a 49 años en 1976, 1982 y 1987 indican que aquellas sin hijos participan más en el mercado de trabajo que las con hijos. No obstante, es im- 
portante destacar otra vez que las mujeres con hijos presentan un importante aumento en la participación económica. Para los años en que se agudiza la recesión económica (1982-1987), la elevación más notable tiene lugar entre las mujeres que tienen 3 hijos y más, siendo el menor de 0 a 3 años.

En países desarrollados las mujeres con hijos pequeños también han incrementado notablemente su participación (véanse Frank Fox y Hesse-Biber, 1984; Roos, 1985). Pero, conviene hacer hincapié en que, a diferencia de esas situaciones, en nuestro país no ha habido modificaciones importantes en la división sexual del trabajo; tampoco existen políticas públicas orientadas explícitamente a reclutar mujeres casadas para actividades específicas, ni ha habido un incremento considerable en la infraestructura de guarderías.

En cambio, el importante descenso en la fecundidad mexicana en el periodo señalado pudo haber liberado tiempo del usualmente dedicado a la crianza de los hijos. ¿Qué evidencia nos ofrecen nuestros datos en este sentido? Más bien nos han llevado a problematizar la posible influencia de la fecundidad sobre el trabajo de las mujeres unidas. Por un lado, se dan aumentos importantes de participación económica entre 1976 y 1987 para mujeres unidas jóvenes (de 20 a 34 años), entre quienes se ha observado el menor descenso de la fecundidad. Este hallazgo restaría poder explicativo al posible impacto del control reproductivo sobre el aumento en la participación económica femenina.

Sin embargo, también tenemos por otro lado el aumento de la presencia económica de las mujeres mayores y con 3 hijos y más en los añós de recesión (1982-1987). Dado que entre ellas se ubican aquellas que controlan su fecundidad, es difícil establecer si los hijos no tenidos le han facilitado su presencia económica, o si en cambio los hijos presentes las han obligado a buscar un sustento adicional.

Durante los años de recesión, también se dan transformaciones sustantivas en la relación entre fecundidad y trabajo entre mujeres pertenecientes a diferentes sectores sociales. Conforme a nuestros resultados, la influencia de los hijos sobre el trabajo femenino depende, en parte, de la dinámica del mercado de trabajo. Así, por ejemplo, en el periodo 1976-1982 que se caracterizó por cierta expansión del mercado de trabajo, incluso las mujeres con hijos chicos de sectores medios y manuales no asalariados, presentaban igual probabilidad de participar económicamente que las mujeres sin hijos. Mientras que en los años de recesión (19821987), la contracción de los empleos femeninos no manuales llevó a reducir la propensión a trabajar de las mujeres con hijos, fren- 
te a las mujeres sin hijos, en los mismos sectores sociales.

En otras situaciones, aun cuando el mercado de trabajo se contrae, las necesidades económicas apremiantes contribuyen a que el número y edad de los hijos reduzcan su papel inhibidor del trabajo extradoméstico de las mujeres. Esto ha ocurrido en 1987 entre los sectores manuales asalariados, donde tradicionalmente los hijos eran un factor altamente limitante de la participación económica femenina; en ese año solamente en situaciones de fuerte carga familiar ( 3 hijos y más con el menor de 0 a 3 años) estas mujeres presentaban menor propensión a desempeñar actividades económicas. Se requiere de un análisis en profundidad de diferentes cohortes de mujeres, pertenecientes a diferentes sectores sociales, para captar el papel que han desempeñado en sus vidas las entradas y salidas del mercado de trabajo en épocas de expansión y crisis económica.

Cambios en la condición femenina: algunos planteamientos de investigación

Hemos documentado hasta aquí los cambios en el trabajo femenino y la fecundidad que han ocurrido en el país en las últimas décadas con base en indicadores agregados provenientes de censos, encuestas de ocupación y encuestas de fecundidad. En este apartado presentamos algunos planteamientos de investigación sobre el significado de estos cambios para la condición de subordinación de las mujeres mexicanas, que se manifiesta en múltiples esferas sociales con distintas intensidades y matices.

En la literatura sobre la génesis y las expresiones actuales de la subordinación de las mujeres se señala la importancia de la división sexual del trabajo intra y extrafamiliar, el control de la sexualidad y de la capacidad reproductiva de las mujeres como factores explicativos de su condición social (Rubin, 1975; Yanagisako, 1979; Harris, 1981; De Barbieri, 1989; Jelin, 1984). El énfasis que se otorga al trabajo y la maternidad se deriva de los argumentos que plantean que no es posible entender plenamente la condición social de las mujeres sin relacionar estrechamente su papel en las esferas de la producción y la reproducción (Benería y Sen, 1982).

Consideramos al igual que otros autores que el análisis de la subordinación femenina y las formas de reproducirla debe hacerse a la par del estudio de su cuestionamiento y alternativas de cambio, mediante el surgimiento de formas de autonomía y resistencia. Bajo esta óptica, la relación de subordinación se reconoce como asimétrica, jerárquica, pero no inmutable, es decir, se conci- 
be como sujeta a transformación. La importancia dada al estudio del cambio en la condición femenina permite hacer visible la creación de espacios de participación que permiten a las mujeres darse cuenta de la subordinación y aislamiento social en que están inmersas y, a su vez, posibilitan respuestas tanto colectivas en la defensa de diversas demandas como individuales, mediante la reorganización de sus proyectos de vida (Ortega, 1985; Massolo y Díaz Ronner, 1985; Acosta, 1985; Acosta et al., 1985; Gerson, 1985; De Barbieri y Oliveira, 1986; Jelin, 1986; Oliveira y Gómez Montes, 1989).

Pero también, varias investigaciones indican que algunos sectores de mujeres no tienen conciencia de las relaciones de subordinación y dominación existentes en el hogar, la consideran como natural; otras aceptan la situación por miedo al abandono y la violencia, y otras más utilizan formas de manipulación más que estrategias de cambio de las relaciones hombre-mujer (Roldán, 1984).

Una manera de captar situaciones de permanencia y cambio en la condición femenina es mediante el análisis del desempeño de diferentes tipos de trabajo, su vinculación con vivencias distintas de la maternidad y la contribución de las diversas situaciones a la transformación de la relación hombre-mujer en el lugar de trabajo y en la familia.

Bajo esta perspectiva, para documentar las diferencias y los matices en la vivencia de la maternidad y del trabajo no es suficiente tener en cuenta situaciones que suponen que los papeles de trabajadora y madre son establecidos de antemano con un grado importante de incompatibilidad entre ellos; éste sería el caso de mujeres que nunca han trabajado y se han dedicado a la maternidad como actividad de tiempo completo, de aquellas que han buscado trabajos de medio tiempo o por cuenta propia para acomodar la actividad económica con la crianza de los hijos, o de situaciones extremas de trabajo que exijan objetiva o subjetivamente la renuncia a los roles maternos. También debe considerarse la existencia de madres trabajadoras que redefinan en algunos aspectos sus roles, tanto de madres como de trabajadoras (véase Hunt, 1984).

Algunos trabajos ya realizados muestran cuán fructífera puede ser esta línea de investigación. Por ejemplo, en un análisis muy sugerente para el caso de Estados Unidos de América, Gerson (1985) estudia un grupo de mujeres que se encuentran en los primeros años de crianza de los hijos, integrantes de una generación que mediante sus decisiones vitales ha contribuido a los cambios en fecundidad y trabajo que tuvieron lugar en ese país en las últi- 
mas décadas. La cohorte elegida es considerada clave para estudiar las causas, consecuencias y el significado de la transformación que está en marcha.

Gerson encuentra 4 patrones generales que los cursos de vida de las mujeres asumen, combinando 2 criterios: las diferencias en cuanto a las expectativas que se tenían al inicio de la vida sobre los planes para la edad adulta y las variaciones en cuanto a las oportunidades y límites que se presentan a lo largo de esta vida adulta. Los tipos son: 1) modelo tradicional: se elige la vida doméstica para la cual se fue preparada en la niñez; 2) modelo de cambio de la domesticidad a la esfera pública: crecientes aspiraciones frente al trabajo y ambivalencia frente a la maternidad; 3) modelo orientado hacia la no domesticidad: las aspiraciones iniciales de no domesticidad fueron reforzadas por las instituciones y experiencias individuales en la vida adulta, y 4) modelo de cambio de la no-domesticidad a la domesticidad: la ambivalencia inicial hacia la maternidad y la vida doméstica es sustituida por aspiraciones decrecientes frente al trabajo.

En otro interesante trabajo para el caso de los sectores populares de Chile, Valdés (1989) se basa en la estrategia de los tipos ideales para reconstruir las articulaciones de sentido con relación a las decisiones de tener o no hijos y dar cuenta de la heterogeneidad que existe entre mujeres que viven en similares condiciones de vida, caracterizadas por la precariedad, la inestabilidad y la pobreza. El primer tipo resultante es denominado como "primacía de lo natural". Lo central aquí es el papel que cumple la mujer en la reproducción de la especie. La mujer debe tener muchos hijos sin limitaciones de ningún tipo. La vida de la mujer que no es madre carece de sentido. El segundo tipo es considerado como "primacía de lo social". Este proyecto tiene por objetivo central la reproducción de la sociedad, con sus estructuras sociales, políticas y culturales así como sus relaciones de poder. La familia adquiere una función económica primordial y la mujer deberá desarrollar nuevas tareas, más allá de las que tiene en el proyecto anterior. Debe ser madre en el contexto de un matrimonio legal y debe ser ama de casa. Por último, tenemos la "primacía de lo individual", el cual es un proyecto que contempla a la mujer como sujeto autónomo, que tiene un plan consciente de desarrollo para su vida que excede a la maternidad. El ser madre se inscribe en un proyecto más amplio que nuede ser vida en pareja, de desarrollo profesional, de participación política y social, de desarrollo artístico.

En suma, estas autoras caracterizan desde situaciones de permanencia de los papeles femeninos tradicionales que plantean a la maternidad como rasgo definitorio de la mujer hasta situacio- 
nes de cambio que requieren la redefinición de los proyectos de vida, el cuestionamiento de la subordinación y la búsqueda de espacios propios. En cierta medida, en ambos casos, los grados de automomía o de subordinación femenina están asociados a factores tales como el contexto de socialización (época, lugar de origen rural o urbano), la edad-experiencia, los recursos sociales y materiales, los tipos de relaciones en que se participa (madre, hija, madre soltera), el grado de estabilidad de la relación de pareja, la aceptación y valoración del compañero y los contenidos culturales aceptados (Gerson, 1985; Valdés, 1989).

\section{Acercamiento metodológico}

Los estudios reseñados tratan de captar el significado que las mujeres otorgan a sus vivencias y la forma en que las incorporan en la organización de sus vidas mediante el análisis microsocial; asimismo hacen hincapié en la construcción y comparación entre tipos sociales que captan la diversidad de proyectos de vida.

Consideramos que, la necesidad analítica de tener en cuenta los puntos de vista de los agentes sociales involucrados lleva a privilegiar la estrategia de análisis microsocial concebida no como una etapa exploratoria de un análisis más global donde serían sometidas a verificación las hipótesis elaboradas, sino más bien como un acercamiento metodológico pertinente en sí mismo por el tipo de conocimiento que se busca. En nuestro caso se trataría de:

a) captar las percepciones de las mujeres sobre los cambios sociales en que han estado involucradas (la ampliación de la participación en el mercado de trabajo, de la escolaridad, el descenso de la fecundidad, entre otras) en épocas de auge y recesión económica;

b) ahondar en el significado de estos cambios para los proyectos de vida, y

c) analizar las estrategias de acción (aceptación, resistencia, negociación, manipulación) que llevan a ratificar o cuestionar las pautas establecidas en torno a la relación entre los géneros en la familia y en el lugar de trabajo.

Es importante hacer especial hincapié en la búsqueda de sentido de estas acciones y en el surgimiento de formas de "darse cuenta", pues esta dimensión es crucial en el logro de los cambios sociales (véase Bourdieu, 1985). Concebimos al individuo como enfrentado a un campo de opciones, ciertamente restringido, pero 
también factible de ser transformado con la acción concreta de los sujetos sociales (véase Przeworski, 1982; Bourdieu, 1985; Jelin, Llovet y Ramos, 1986; Gerson, 1985; Valdés, 1989).

Este acercamiento es distinto al reportado en la primera parte de este trabajo donde se establecieron tendencias sobre cambios en el trabajo de las mujeres y su fecundidad, y es por esto que se recurrió al análisis de encuestas nacionales y censos de población. En la estrategia microsocial interesa llevar a cabo análisis plausibles sobre el significado que tienen los diversos procesos estudiados para las vidas cambiantes de distintos tipos de mujeres involucradas (véase Caldwell, 1988). Consideramos ambas estrategias complementarias y compartimos la necesidad expresada por numerosos autores de combinar en una misma investigación distintos estilos de trabajo y herramientas de investigación. Desde nuestro punto de vista, la investigación social avanza transitando múltiples caminos, y el reto consiste en buscar los puntos de encuentro a la vez que establecer la pertinencia de cada uno.

Partiendo de las premisas anteriores, el análisis microsocial de las vivencias de la maternidad y del trabajo debe privilegiar una perspectiva comparativa entre mujeres de diferentes regiones, grupos sociales y cohortes que estén involucradas en distintas situaciones de trabajo.

En países como México, de grandes desigualdades económicas, sociales, geográficas y culturales, es relevante tener en cuenta mujeres de diferentes grupos sociales que estén ubicadas en distintas regiones del país como forma de acercarse a diversas estructuras de oportunidades y ambientes culturales. Asimismo, es necesario incorporar de manera explícita los escenarios de la casa y el lugar de trabajo. Como es ampliamente conocido, las pautas más usuales de reproducción de la población se dan al interior de unidades domésticas; esto es, presuponen la formación de familias y su organización en arreglos residenciales funcionales a la reproducción de la vida cotidiana. Por su parte, la participación o no participación en contextos laborales que se ubican espacialmente fuera de los ámbitos familiares establece para las mujeres, en principio, el rango de posibilidades para romper el aislamiento del hogar y ampliar su ámbito de relaciones y participación social. $^{2}$

Por último, la comparación entre cohortes permite analizar los cambios en la condición social de sectores de mujeres que han

\footnotetext{
${ }^{2}$ Para una fundamentación de la relevancia de ubicar a los individuos en diferentes contextos sociales (espaciales, familiares, laborales) véanse entre otros: Oliveira y García, 1986; Oliveira y Salles, 1988; Jelin, Llovet y Ramos, 1986).
} 
vivido eventos cruciales en su vida (transiciones) en diferentes momentos de expansión y crisis de la economía y la sociedad. Se busca privilegiar el estudio de transiciones en el ámbito individual, familiar y laboral: entrada al mercado de trabajo, migración, unión, nacimiento de los hijos, inicio de la participación en organizaciones sindicales, comunitarias y políticas, que pueden o no traer rupturas en los patrones tradicionalmente aceptados. ${ }^{3}$ Entre los aspectos a considerar en cada transición están, entre otros: la participación activa en la toma de decisiones, las razones tenidas en cuenta, los cambios desencadenados en las diversas esferas de la vida, la percepción de conflictos, el surgimiento de formas de resistencia y acomodo, los cambios percibidos en las relaciones hombre-mujer y el significado del evento en la vida de las mujeres.

\section{Algunas hipótesis a modo de conclusión}

La investigación realizada en México sobre las repercusiones del trabajo de las mujeres sobre su condición social, al igual que lo que ocurre en otras realidades nacionales, ha estado principalmente encaminada a refutar las hipótesis planteadas en los años sesenta y setenta a partir de ciertas interpretaciones de la teoría marxista que afirmaban que el hecho de trabajar en sí llevaba a un cambio en la condición de la mujer. Es común que los estudios lleguen a la conclusión de que la incorporación de las mujeres al mercado de trabajo no les permite replantear de manera significativa la relación entre los géneros. ${ }^{4}$

Estar de acuerdo con los planteamientos anteriores por supuesto no implica negar que están ocurriendo cambios de diversa índole en la condición femenina en nuestra sociedad, muchas veces evidenciados a través de ajustes, acomodos, o replanteamientos en algunas áreas específicas, que sólo pueden ser conocidas con mayor profundidad mediante la investigación concreta. $\mathrm{Mu}-$ chas veces estas prácticas quedan oscurecidas por la necesidad que existe en los diversos tipos de estudios de documentar la permanencia esencial de la subordinación de las mujeres.

Como hemos anticipado, el cambio tiene que ser buscado mediante una estrategia comparativa que tome en cuenta los tiempos

\footnotetext{
${ }^{3}$ Más elementos sobre esta estrategia se encuentran en Jelin y Feijóo, 1983; Eider, 1978; Hareven, 1978, entre otros.

4 Véanse, por ejemplo, los trabajos reunidos en el libro Trabajo, poder y sexualidad, coordinado por Oliveira, O., 1989a.
} 
diferenciales vividos por cada cohorte de madres. Además es crucial incorporar el tipo de trabajo que se desempeña, el cual se encuentra altamente relacionado con el sector social de pertenencia. El significado del trabajo para las vidas de las madres seguramente será muy distinto de acuerdo con el ţipo de actividad que se desempeña.

En el caso de las actividades por cuenta propia que se realizan en el hogar a la par del cuidado de la casa y de los hijos, y donde las mujeres mayores tienen un lugar destacado, puede haber un aumento de la carga de trabajo y un reforzamiento de los patrones tradicionales de división intrafamiliar del mismo.

Sin embargo, aun en estas situaciones no existe una relación sencilla entre el hecho de que las esposas tengan una fuente independiente de ingresos y las pautas de interacción matrimonial y familiar, como han documentado Benería y Roldán (1987) para el caso de los sectores populares de la ciudad de México. Estas autoras no encontraron que el control femenino sobre algún ingreso les permitió negociar de manera significativa las relaciones entre los géneros al interior de los hogares. Sin embargo, documentan diferencias de cierta importancia según la magnitud del ingreso que aportan las mujeres. En los casos en que este aporte es crucial para la manutención del hogar ellas tienen cierto éxito en el logro de un mayor respeto y buen trato. Asimismo, establecen de manera general que los salarios femeninos, no importa cuán bajos, pueden ser usados como una palanca para asegurar un espacio mínimo de control autónomo, como un mecanismo para alcanzar mejores niveles de vida, y para atenuar el daño a la autoimagen causado por la dependencia económica de sus compañeros.

La situación puede ser distinta si se trata de un trabajo asalariado, donde las mujeres más jóvenes tienen mayor espacio, aunque sujeto a cambios como vimos con anterioridad. Por la naturaleza de este tipo de trabajo, éste puede implicar alternativas de cambio relacionadas con la valoración de la mujer en el lugar de trabajo, la creación de espacios de autonomía que rompen el aislamiento del hogar, el logro de satisfacción con la actividad realizada, la posibilidad de participación en actividades sindicales y cierta independencia económica. Sin embargo, la división sexual del trabajo prevaleciente en los mercados laborales, que se manifiesta en la ubicación de las mujeres en ocupaciones "femeninas" que pagan salarios bajos, de manera frecuente no les da acceso a posibilidades de promoción y movilidad social que les permitan organizar sus vidas alrededor de otros ejes además de la maternidad.

A pesar de estas limitaciones, el trabajo asalariado, sobre todo 
el de tiempo completo, puede traer una reorganización importante de la vida cotidiana de las mujeres casadas al tener que delegar en otras personas, al menos parcialmente, las tareas de la casa y el cuidado de los hijos. Entre las estrategias utilizadas para combinar maternidad y trabajo en México y otros países están. la utilización de servicio doméstico remunerado entre sectores medios, la activación de redes sociales de apoyo entre vecinos, parientes y amigos y la utilización de guarderías entre diferentes sectores sociales. En menor medida podemos tener la reorganización de la división del trabajo en la casa, aspecto que implica negociaciones o acomodos diferentes en la relación hombre-mujer.

Otro aspecto importante a considerar es el grado de compromiso con el trabajo. Es factible suponer que cuando se asumen distintos tipos de trabajo (por cuenta propia o asalariados; manuales y no manuales) como carrera, se incrementan las posibilidades de cuestionar y redefinir la valorización de la maternidad como el atributo esencial del ser mujer y la dedicación exclusiva de las mujeres a los trabajos reproductivos (procreación, cuidado y socialización de los hijos y tareas domésticas de manutención cotidiana).

Por su misma naturaleza, el trabajo como carrera ha sido mayormente estudiado en los países desarrollados. Como destaca Gerson (1985) el cuestionamiento de los valores y normas tradicionales que asignan a las mujeres el papel de madres de tiempo completo o suponen que las mujeres con carreras no deben tener hijos abren alternativas de redefinición de la maternidad y el trabajo como actividades que no son mutuamente excluyentes. Las posibilidades de combinar maternidad y trabajo requieren de modificaciones importantes en ideas tradicionalmente aceptadas de que los hijos sufren con la ausencia de la madre, de que las mujeres deben optar entre una carrera exitosa y los hijos. Asimismo, presupone en gran parte de los casos la existencia de una relación de pareja estable y una actitud del compañero de valoración del trabajo de la mujer, de percepción de la importancia de su contribución económica para la manutención de la familia y de disposición de ayudar en el cuidado de la casa y crianza de los hijos. Hipotetizamos que estas transformaciones todavía no se han dado en la sociedad mexicana en forma notable; pero consideramos, al igual que Gerson (1985), que el establecimiento de compromisos con la maternidad y el trabajo abro una alternativa de cambios en el papel de las mujeres, en las relaciones sociales entre géneros, y por ende en la condición de subordinación de la población femenina. 


\section{Bibliografía}

Acosta, Mariclaire et al. (1985). "La respuesta de las mujeres frente al militarismo y la represión en América Latina", documento presentado en el Taller de Investigación Social sobre la Mujer, México, El Colegio de México.

(1985). "Prácticas represivas y sus efectos en la cultura política: algunas reflexiones", documento presentado en el Taller de Investigación Social sobre la Mujer, México, El Colegio de México.

Benería, Lourdes y Marta Roldán (1987). The Crossroads of Class and Gender (Industrial Homework, Subcontracting and Household Dynamics in Mexico Cityl, Chicago, The University of Chicago Press.

y Gita Sen (1982). "Acumulación, reproducción y el papel de la mujer en el desarrollo económico: una revisión a Boserup", en Magdalena León et al., Las trabajadoras en el agro, Colombia, ACEP, pp. 23-39.

Blau, Francine D. y Marianne A. Ferber (1985). "Women in the Labor Market: the last twenty years", en Laurie Larwood et al., Women and Work, Nueva York, Sage Publications, pp. 19-49.

Bourdieu, Pierre (1985). "The Social Space and the Genesis of Groups", en Social Science Information, vol. 24, núm. 2, Londres, Beverly Hills y Nueva Delhi, SAGE.

Bronfman, Mario, Elsa López y Rodolfo Tuirán (1986). "Práctica anticonceptiva y clases sociales en México: la experiencia reciente", en Estudios Demográficos y Urbanos, vol. 1, núm. 2, México, El Colegio de México, pp. 165-203.

Caldwell, John C. (1988). "Micro Approaches: Similarities and Differences, Strengths and Weaknesses", en John Caldwell, Allan Hill y Valerie Hill, Micro-Approaches to Demographic Research, Londres, Kegan \& Paul International.

Casar, Jos y Jaime Ros (1987). "Empleo, desempleo y distribución del ingreso", en Carlos Tello (coord.), México: informe sobre la crisis 19821986, México, UNAM.

Cervantes, Alejandro y Germán Sandoval (1988). "Regulación de la fecundidad", en Encuesta Nacional sobre Fecundidad y Salud: 1987, Memoria de la Reunión celebrada el 30 de septiembre, Secretaría de Salud, pp. 133-149.

Cortés, Fernando (1988). "El mercado de trabajo urbano y la sociodemografía mexicana en la mitad de la década de los ochenta: algunas consideraciones metodológicas", en Memorias de la III Reunión Nacional sobre Investigación Demográfica en México, México, Sociedad Mexicana de Demografía/unam.

De Barbieri, Teresita (1989). “Trabajos de la reproducción”, en Orlandina de Oliveira, Marielle Pepin Lehalleur y Vania Salles (comps.), Grupos domésticos y reproducción cotidiana, México, unam/Miguel Ángel Porrúa/El Colegio de México, pp. 11-37. y Orlandina de Oliveira (1986). “Nuevos sujetos sociales: la pre- 
sencia política de las mujeres en América Latina", en Nueva Antropología, vol. VIL núm. 30, México.

Elder Jr., G.H. (1978). "Family History and the Life Course", en T. Hareven (ed.), Transitions: The Family and the Life Course in Historical Perspective, Nueva York, Academic Press.

Encuesta Mexicana de Fecundidad (1979). México, Primer Informe Nacional, México, SPP-IISUNAM.

Encuesta Nacional Demográfica (END) (1982). México (CONAPO).

Encuesta Nacional de Fecundidad y Salud (ENFES) (1987). Memoria de la reunión celebrada el 18 de febrero de 1987, México, Dirección General de Planificación Familiar, Secretaría de Salud.

Frank Fox, Mary y Sharlene Hesse-Biber (1984). Women at Work, Mayfield Publishing Company.

García, Brígida (1987). "Cambio demográfico y niveles de vida", en Estudios Demográficos y Urbanos, vol. 2, núm. 2, México, El Colegio de México.

y Orlandina de Oliveira (1989). "The Effect of Variation and Change in Female Economic Roles upon Fertility Change in Developing Countries", ponencia presentada en la XxI Conference of the International Union for the Scientific Study of Population, Nueva Delhi.

y Orlandina de Oliveira (1990a). "Cambios en la presencia femenina en el mercado de trabajo: (1976-1987)", México, El Colegio de México (mimeo.).

y Orlandina de Oliveira (1990b). "Recesión económica y cambio en los determinantes del trabajo femenino", México, El Colegio de México (mimeo.).

García y Garma, Irma O. (1989). "La fecundidad en las áreas rurales y urbanas de México", en Estudios Demográficos y Urbanos, vol. 4, núm. 1, México, El Colegio de México.

Gerson, Kathleen (1985). Hard Choices. How Women Decide about Work, Career, and Motherhood, University of California Press.

González de la Rocha, Mercedes (1989). "Crisis, economía doméstica y trabajo femenino en Guadalajara”, en Orlandina de Oliveira (coord.), Trabajo, poder y sexualidad, México, pp. 159-189.

Harris, Olivia (1981). "Household as Natural Units", en Kate Young, Carol Wolkowitz y Roslyn McCullagh (comps.), Of Marriage and the Market: Women's Subordination in Internationa: Perspective, Londres, CSE Books, pp. 75-107.

Hareven, T. (1978). Transitions: The Family and the Life Course in Historial Perspective, Nueva York, Academic Press.

Hunt, J. (1984). "Development of Rapport Through the Negotiation of Gender in Field Work Among Police", en Human Organization, pp. 96283.

Jelin, Elizabeth y María del Carmen Feijóo (1983). "Presiones cruzadas: trabajo y familia en la vida de las mujeres", en Catalina Wainerman, Elizabeth Jelin y María del Carmen Feijóo, Del deber ser y el hacer de las mujeres. Dos estudios de caso en Argentina, México, PISPAL-El Colegio de México. 
(1984). Familia y unidad doméstica: mundo público y vida privada, Buenos Aires, Estudios CEDEs.

(1986a). Ciudadanía e identidad. La mujer en los movimientos sociales en América Latina, Buenos Aires, CEDES.

Juan José Llovet y Silvina Ramos (1986b). "Un estilo de trabajo: la investigación microsocial", en Rodolfo Corona et al., Problemas metodológicos en la investigación sociodemográfica, México, PISPAL-EI Colegio de México.

López, Elsa y Rodolfo Tuirán (1983). "La fecundidad y los grupos sociales en México: nuevos datos y nuevas hipótesis", ponencia presentada en el X Congreso Mundial de Sociología, agosto.

Lustig, Nora (1986). "Economic Crisis and Living Standards in Mexico: 1982-1985", documento preparado para el proyecto sobre The Impact of Global Recession on Living Standars in Selected Developing Countries, organizado por UNDP.

Massolo, Alejandra y Lucila Díaz Ronner (1985). "Consumo y lucha urbana en la ciudad de México: mujeres protagonistas" (mimeo.).

Mier y Terán, M. y C. Rabell Romero (1984). "Fecundidad y grupos sociales en México (1971-1977)", en Los factores del cambio demográfico en México, México, Siglo XXI-IISUnAm, pp. 221-224.

Naciones Unidas (1987). Women's Employment and Fertility, Fertility Behaviour in the Context of Development. Evidence from the World Fertility Survey, Nueva York.

Oliveira, Orlandina de y Brígida García (1986). "Encuestas. ¿Hasta dónde?", en Rodolfo Corona et al., Problemas metodológicos en la investigación sociodemográfica, México, PISPAL-El Colegio de México. y Vania Salles (1988). "Reflexiones teóricas para el estudio de la reproducción de la fuerza de trabajo", en Argumentos, núm. 4, México, Universidad Autónoma Metropolitana-Xochimilco, pp. 19-45. (1989a). "Empleo femenino en México en tiempos de recesión económica: tendencias recientes", en Jennifer Cooper, Teresita de Barbieri, Teresa Rendón, Estela Suárez y Esperanza Tuñón (comps.), Fuerza de trabajo femenina urbana en México, México, unam y Miguel Ángel Porrúa.

(1989b) (coord.). Trabajo, poder y sexualidad, México, PIEM-El Colegio de México.

y Liliana Gómez Montes (1989c). "Subordinación y resistencia femeninas: notas de lectura", en Orlandina de Oliveira (coord.), Trabajo, poder y sexualidad, México, PIEM-El Colegio de México.

y Brígida García (1990). "Expansión del trabajo femenino y transformación social en México: 1950-1987", en México en el umbral del milenio, México, CEs-El Colegio de México, pp. 345-374.

Ortega, Adriana (1985a). "La no violencia iuna alternativa para nosotras?", en Fem, año 8, núm. 37, pp. 55-57.

(1985b). "La respuesta delas mujeres frente a la represión y el militarismo. El caso de México", documento presentado en el seminario, La participación social de la mujer, diciembre, México, El Colegio de México. 
Pacheco Gómez Muñoz, María Edith (1988). Población económicamente activa femenina en algunas áreas urbanas de México en 1986, tesis de maestría, México, CEDDu, El Colegio de México.

Palma, Yolanda, (1988). "Niveles de la fecundidad", en Encuesta Nacional sobre Fecundidad y Salud: 1987, Memoria de la reunión celebrada el 30 de septiembre de 1988, México, Dirección General de Planificación Familiar de la Secretaría de Salud, pp. 79-92.

Pedrero Nieto, Mercedes (1990). "Cambios en la actividad económica femenina y la transición de la fecundidad en zonas metropolitanas", ponencia presentada en la IV Reunión Nacional sobre Investigación Demográfica en México, abril.

y Teresa Rendón (1982). "El trabajo de la mujer en México en los setentas", en Estudios sobre la mujer 1. Empleo y la mujer. Bases teóricas, metodología y evidencia empírica, México, INECI-SPP, Serie Lecturas nI.

Przeworski, A. (1982). "Teoría sociológica y el estudio de la población: reflexiones sobre el trabajo de la Comisión de Población y Desarrollo de CLACSO", en Reflexiones teórico-metodológicas sobre las investigaciones en población, México, El Colegio de México, pp. 58-99.

Rendón, Teresa y Carlos Salas (1987). "Evolución del empleo en México: 1895-1970", en Estudios Demográficos y Urbanos, vol. 2, núm. 2, México, El Colegio de México, pp. 189-230.

Roldán, Marta (1984). "Pautas de control del circuito monetario doméstico y formas de conciencia entre trabajadoras industriales domiciliarias en la Ciudad de México", ponencia presentada en la Reunión de Investigación sobre la Mujer e Investigación Feminista: Balances y Perspectivas de la Década de la Mujer en América Latina, del 8 al 11 de diciembre, Montevideo, Uruguay, GRECMU.

Roos, Patricia A. (1985). Gender and Work: A Comparative Analysis of Industrial Societies, Nueva York, State University of New York Press.

Rubin, Gayle (1975). "The Traffic of Women", en Reiter Reyna, Anthropology of Women, Nueva York, Monthly Review Press.

Stathan, Anne, Eleanor M. Miller y Hans O. Mauksch (1988). The Worth of Women's Work, Nueva York, New York University Press.

Selva, Beatriz (1985). Modalidades de trabajo femenino en San Felipe del Agua, México, FlACSO, serie de tesis de maestría.

Tello, Carlos (1979). La política económica en México, México, Siglo XXI. (1987). "Introducción" en Carlos Tello (coord.), México: informe sobre la crisis 1982-1986, México, UNAM.

Valdés, Teresa (1989). Venid, benditas de mi padre, Santiago de Chile, FLACSO.

Wainerman, Catalina y Zulma Recchini de Lattes (1981). El trabajo femenino en el banquillo de los acusados. La medición censal en América Latina, México, Terranova y Population Council.

Yanagisako, Sylvia (1979). "Family and Household: The Analysis of Domestic Groups", Annual Review of Anthropology, vol. 8, Stanford, Department of Anthropology, Stanford University. 